\title{
Impact of Suture Conchopexy on Olfaction and the Risk of Middle Turbinate Lateralization
}

\author{
Ibrahim Sumaily $^{1}$, Ibrahim Alarifi ${ }^{1}$, Labeb M. Sailan ${ }^{1}$, Saad Alsaleh ${ }^{2}$, Mohammad Aloulah ${ }^{1}$ \\ 1. Otorhinolaryngology, King Abdulaziz University Hospital, Riyadh, SAU 2. Otorhinolaryngology, King Saud \\ University, Riyadh, SAU
}

Corresponding author: Ibrahim Sumaily, sumaily.i@gmail.com

\begin{abstract}
Introduction: Middle turbinate (MT) lateralization is one of the common causes of endoscopic sinus surgery (ESS) failure and often necessitate revision surgery. To avoid this sequala, surgeons have attempted several methods to keep the MT medialized. One such method is conchopexy. However, the impact of this procedure on olfaction remains unclear.
\end{abstract}

Method: A retrospective cohort study was conducted to compare the subjective olfaction outcome of ESS in patients for whom conchopexy was performed and in controls where a spacer was applied in the middle meatus. Also, the risk of lateralization in both techniques was compared. In addition, other factors related to the outcome of olfaction, such as age, gender, type of chronic rhinosinusitis (CRS), and partial resection of the MT, were assessed.

Results: Out of 299 patients with CRS who underwent ESS, 134 met our inclusion criteria. In total, 62.7\% were male and $37.3 \%$ were female, and their mean age was 37.4 years. Sixty-one patients (cases) underwent conchopexy, and 73 patients (controls) underwent insertion of a middle meatus spacer. None of the subjects in both groups developed anosmia or hyposmia as a complication. The improvement of olfaction was almost equal in both groups (for anosmia: $92.9 \%$ in cases vs. $87.5 \%$ in control; for hyposmia $87.1 \%$ in cases vs. $89.7 \%$ in control). In patients with anosmia, the improvement of olfaction was lower when the MT was partially resected (71.4\% vs. $95.7 \%)$; whereas, in patients with hyposmia, the improvement was not significantly different ( $87 \%$ vs. $93.8 \%$ when the MT was partially resected). The improvement of olfaction was higher in patients with allergic fungal sinusitis (AFS) and CRS with nasal polyps (CRSwNP) than in those with CRS without nasal polyps (CRSsNP). The MT lateralization was almost equal in both groups ( $9.0 \%$ in cases vs. $9.6 \%$ in controls).

Conclusion: Conchopexy does not affect olfaction subjectively. The improvement of olfaction is related more to the underlying disease, i.e., less improvement occurs in cases of CRSsNP. The risk of lateralization is equal with either conchopexy or middle meatus spacer.

Received 09/25/2019

Review began 09/28/2019 Review ended 09/29/2019 Published 10/01/2019

๑) Copyright 2019

Sumaily et al. This is an open access article distributed under the terms of the Creative Commons Attribution License CC-BY 3.0., which permits unrestricted use, distribution, and reproduction in any medium, provided the original author and source are credited.
Categories: Otolaryngology

Keywords: keywords: conchopexy, middle turbinate, lateralization, endoscopic, sinus

\section{Introduction}

The middle turbinate (MT) is an important surgical landmark in functional endoscopic sinus surgery (FESS) [1]. Postoperatively, lateralization of the MT with scarring and obstruction of the middle meatus after endoscopic ethmoidectomy has accounted for a high percentage of postoperative complications [2-5]. However, some published reports have mentioned that it is the most common complication of endoscopic sinus surgery and often necessitate a revision surgery [5-6]. Turbinate medialization techniques have gained popularity, as its main aim is to prevent turbinate lateralization [3,7]. Theoretically, adhesions between the septum and MT prevent lateralization but may compromise airflow to the olfactory neuroepithelium and affect the sense of olfaction [3].

MT medialization without resection is an approach designed to both preserve the MT and prevent lateralization, which may cause obstruction of the airflow in the ethmoid, maxillary, and frontal sinuses after endoscopic sinus surgery [8].

Numerous methods have been used to assist in the avoidance of this complication including the use of packing in the ethmoid sinus as a "spacer", creation of synechia between the MT and septum (bolgerization), and suture medialization of the MT to the septum (conchopexy) [5,9]. While the conchopexy is an effective technique, because the olfactory groove lies superior in the groove between the MT and septum, concerns have been raised as to the effect of this maneuver on olfaction [9]. The long-term results of these techniques are lacking [10]. The conchopexy technique has been criticized for being technically difficult and timeconsuming, mainly due to the need to tie a knot within the nasal cavity; therefore, another technique called non-knot conchopexy is applied by using double through and through sutures without applying a knot to the 
In our center, either the conchopexy or middle meatus spacer technique is used for almost every patient. In this study, we aimed to assess the risk for olfaction loss in patients who undergo conchopexy and middle meatus packing and to determine the incidence of MT lateralization in these two groups.

\section{Materials And Methods}

This retrospective cohort study was conducted in our tertiary hospital. After obtaining the approval of the institutional review board to conduct this study, we reviewed all of the patients who underwent endoscopic sinus surgery with either middle meatus packing or suture conchopexy for chronic rhinosinusitis (CRS) and followed up for at least six months. We excluded those who underwent revision surgery, those who underwent complete resection of the MT, those with benign or malignant tumors and those with incomplete records or missing data. We reviewed olfaction status pre- and post-operatively, and the position of the MT postoperatively. Other variables studied include age, gender, type of CRS, surgical procedures done for the middle turbinate, septoplasty, inferior turbinate intervention, use of steroids postoperatively, patency of the nasal airways pre- and postoperatively, patency of the sinus ostia postoperatively. Subjects were divided into two groups; the first group is those who underwent endoscopic sinus surgery with their MTs kept medialized with suture conchopexy to the septum (cases), and the second group are those whose MTs were kept medialized through the insertion of a spacer in the middle meatus, NasoPore (controls). We focused mainly on the subjective olfaction outcome in these two groups and the factors associated with this outcome according to their follow up assessment records between six months to one year. The preoperative status of olfaction was normal olfaction, hyposmia, or anosmia. The postoperative status of olfaction was normal olfaction as preoperative status, improved hyposmia or anosmia, non-improved hyposmia or anosmia, or new onset of hyposmia or anosmia. This assessment is done at the follow-up visit between six and 12 months postoperatively. We used SPSS software, version 22 (SPSS Inc., Chicago, IL) for data analysis.

\section{Results}

In total, 299 patients with CRS who underwent ESS between January 2015 and December 2017 were enrolled in this study. Of them, 134 met our inclusion criteria. The other 165 patients were excluded for the following reasons: 78 patients had undergone surgery previously, 22 had tumors, 16 had undergone complete resection of the MT, and 49 had incomplete records. Patient age ranged between 14 and 80 years, and the mean age was 37.4 years, with a standard deviation of 13.8 years. In total, $62.7 \%$ were male and $37.3 \%$ were female. Preoperatively, $87.3 \%$ had partial nasal obstruction, $9.0 \%$ had complete nasal obstruction, and the remaining 3.7\% had no nasal obstruction. Of those patients with nasal obstruction, $79.9 \%$ had bilateral nasal obstruction. Preoperatively, $52.2 \%$ had hyposmia, $22.4 \%$ had anosmia, and the rest (25.4\%) had normal olfaction. There was no gender difference in the preoperative status of olfaction. All those with no nasal obstruction were having normal olfaction. The most prevalent disease type was CRS with polyps (CRSwNP) (64.9\%), followed by CRS without nasal polyps (CRSsNP) (27.6\%) and allergic fungal sinusitis (AFS) (7.5\%). The prevalence of CRSwNP was higher in males, whereas the prevalence of CRSsNP and AFS were higher in females. The prevalence of AFS in females was four times that in males. In total, $98.5 \%$ had bilateral disease. All those with complete obstruction had CRSwNP, and bilateral nasal obstruction was higher in patients with CRSwNP. In addition, patients with CRSwNP always had bilateral disease. Olfaction was less affected in patients with CRSsNP (43.2\%) as seen in Table 1 . The MTs were partially resected in $22.4 \%$ of the cases either because of polypoidal mucosa or concha bullosa. The remaining patients did not undergo MT reduction.

Suture conchopexy was done for 61 patients (cases), and the middle meatus spacer was used for 73 patients (controls). None of the subjects in both groups, who have normal olfaction preoperatively (34 candidates), developed new-onset anosmia or hyposmia as a complication after surgery. Overall olfaction improvement occurred in $89 \%$. The conchopexy showed no negative impact on the olfaction improvement (88.9\% vs. $89.1 \%$ in the middle meatus group, $\mathrm{P}$-value=0.975). This was not statistically affected by the age or the gender of the candidates. In patients with anosmia, the improvement of olfaction was lower when the MT was partially resected (71.4\% vs. $95.7 \%$ ), whereas, in patients with hyposmia, the improvement was slightly higher when the MT was partially resected (87\% vs. 93.8\%) (Tables $1-2)$. The improvement of olfaction was higher in patients with AFS than in patients with CRSwNP or those with CRSsNP $(100 \%, 89.5 \%$, and $81 \%$, respectively). Systemic steroid administration postoperatively was associated with higher improvement of olfaction among subjects, but not statistically significant (p-value 0.269). 


\section{Cureus}

\begin{tabular}{|c|c|c|c|}
\hline Variable & Spacer & Conchopexy & $P$ value \\
\hline $\mathrm{N}$ & 73 & 61 & \\
\hline Age & $35.8(+14.8)$ & $39.4(+12.2)$ & 0.136 \\
\hline Gender (male) & $53.4 \%$ & $73.8 \%$ & 0.015 \\
\hline Preoperative nasal obstruction (partial) & $82.2 \%$ & $93.4 \%$ & 0.563 \\
\hline Preoperative hyposmia or anosmia & $75.3 \%$ & $73.8 \%$ & 0.964 \\
\hline Diagnosis (CRSwNP, CRSsNP, AFRS) & $57.5 \%, 34.2 \%, 8.2 \%$ & $73.8 \%, 19.7,6.6$ & 0.102 \\
\hline Partial resection of the MT & $26.0 \%$ & $18.0 \%$ & 0.272 \\
\hline Septoplasty & $73.1 \%$ & $66.7 \%$ & 0.610 \\
\hline Inferior turbinate reduction & $78.8 \%$ & $61.1 \%$ & 0.142 \\
\hline Olfaction improvement Postoperatively & $89.1 \%$ & $88.9 \%$ & 0.975 \\
\hline Postoperative systemic steroid administration & $60.3 \%$ & $68.9 \%$ & 0.306 \\
\hline Postoperative nasal obstruction (subjective) & $4.1 \%$ & $0.0 \%$ & 0.111 \\
\hline Postoperative nasal airway (patent) & $98.6 \%$ & $100 \%$ & 0.363 \\
\hline Middle turbinate lateralization & $9.6 \%$ & $9.0 \%$ & 0.825 \\
\hline
\end{tabular}

TABLE 1: Comparison between the suture conchopexy and middle meatus spacer groups

MT: Middle turbinate, CRSwNP: Chronic rhinosinusitis with nasal polyposis, CRSsNP: Chronic rhinosinusitis without polyposis, AFS: Allergic fungal sinusitis. 


\section{Cureus}

\begin{tabular}{|c|c|c|c|}
\hline Variable & Improved olfaction & Not improved & $P$ value \\
\hline $\mathrm{N}$ & 89 & 11 & \\
\hline Age & $35.84(+13.2)$ & $41(+12.57)$ & 0.224 \\
\hline Gender (male) & $61.8 \%$ & $54.5 \%$ & 0.646 \\
\hline Preoperative nasal obstruction (partial) & $87.6 \%$ & $90.9 \%$ & 0.756 \\
\hline Preoperative olfaction status (hyposmia or anosmia) & $100 \%$ & $100 \%$ & 1.000 \\
\hline Diagnosis (CRSwNP, CRSsNP, AFRS) & $76.4 \%, 14.6 \%, 9 \%$ & $72.7 \%, 27.3,0.0$ & 0.789 \\
\hline Partial resection of the MT & $22.5 \%$ & $27.3 \%$ & 0.724 \\
\hline Septoplasty & $73.5 \%$ & $75 \%$ & 0.951 \\
\hline Inferior turbinate reduction & $73.5 \%$ & $75 \%$ & 0.951 \\
\hline Suture Conchopexy & $44.9 \%$ & $45.5 \%$ & 0.975 \\
\hline Postoperative systemic steroid & $78.7 \%$ & $63.6 \%$ & 0.269 \\
\hline Postoperative nasal obstruction (subjective) & $0.0 \%$ & $18.2 \%$ & 0.001 \\
\hline Postoperative nasal airway (patent) & $98.9 \%$ & $100 \%$ & 0.727 \\
\hline Middle turbinate lateralization & $11.2 \%$ & $9.1 \%$ & 0.688 \\
\hline
\end{tabular}

TABLE 2: Comparison between cases of improved olfaction postoperatively and non-improved olfaction postoperatively groups

MT: Middle turbinate, CRSwNP: Chronic rhinosinusitis with nasal polyposis, CRSsNP: Chronic rhinosinusitis without polyposis, AFS: Allergic fungal sinusitis.

\section{Discussion}

As part of endoscopic sinus surgery, the MT is almost always medialized to perform middle meatus antrostomy, ethmoidotomy, and frontal sinusotomy. The MT can lateralize postoperatively, and several techniques to prevent lateralization of the MT and its sequelae have been described and discussed in the literature, such as controlled synechia (bolgerization) [12], insertion of metallic clips between the head of the MT and septum [1], suture conchopexy [2], and synechia with bovine serum albumin tissue adhesive [8].

Medialization techniques are theoretically expected to interfere with olfaction. In our study, we did not note a significant difference in the subjective olfaction outcome between the suture conchopexy and middle meatus spacer groups, and this result is in line with the findings of the prospective controlled study by Friedman et al. that compared olfaction before and after MT medialization [3]. In addition, Dutton et al. who conducted a prospective non-controlled study of patients who underwent functional endoscopic sinus surgery with suture medialization of the MT reported a small but significant improvement in olfactory function after MT suture medialization to the septum compared with the preoperative status, as assessed using the University of Pennsylvania Smell Identification Test (UPSIT) [9]. Hegazy et al. who compared on 39 patients the olfaction outcome of non-intervention, bolgerization, and suture conchopexy groups reported no significant improvement of olfaction in all the three groups [13].

In our study, we found that the incidence of lateralization in those who underwent suture conchopexy was almost equal to that in patients who underwent insertion of a spacer in the middle meatus, and this number is higher than what Thorton RS mentioned in his retrospective study (9\% vs. 1.67\%) [2]. This number is also higher than the incidence of lateralization noted when using controlled synechia, as reported by Friedman et al. who used the microdebrider to induce synechia between the MT and septum [6]. The incidence of lateralization in the present study is slightly lower than what Hewitt and Orlandi reported through their retrospective study; in their study, adhesions between the MT and lateral nasal wall developed in $10.8 \%$, but most of these adhesions were easily released in the clinic [7]. In a prospective study by Dutton et al. on suture conchopexy in endoscopic sinus surgery cases, postoperative endoscopic examination revealed that lateralization of the MT was a rare complication after suture medialization of the MT [9]. But in this study no control group to compare.

There were no specific olfaction outcome tool results available in the patient records. Actually, there is no 
validated Arabic version of UPSIT. Although we used the subjective assessment of the olfaction status pre and postoperatively, it is very unlikely to fall in the estimation bias of the visual analogue scale, which can be affected by the outcome of the other symptoms. Also, for this reason, we excluded 49 of our candidates because of the incomplete or unclear records of their olfaction status either pre- or postoperatively.

The combination of both suture conchopexy and middle meatus spacer insertion was studied by Chen et al. in their prospective, randomized, blind controlled study; they found that the combination was associated with a significantly lower incidence of lateralization as well as synechia formation between the MT and lateral nasal wall [14].

In addition, Hanna and Kilty compared the cost-effectiveness of suture medialization and middle meatus stenting in 60 cases. They found that the commercial stent use cost ranged from 8 to 83 times the price of the suture depending on the stent [15].

Regarding the risk for disease recurrence and the need for revision surgery, Bofares, in his controlled trial involving four groups, namely, the medialization only, partial MT resection, medialization with controlled synechia, and septo-turbinal suture groups, found that medialization only was associated with $49 \%$ recurrence of sinusitis, partial resection was associated with $12 \%$ recurrence, and in the other two groups, there was no recurrence [16].

Our study is limited by being retrospective study and by using subjective olfaction status, not the UPSIT. However, UPSIT is also a subjective test and can confuse between normosmia and hyposmia as it is affected by the educational and cultural background of the candidate as several studies showed. No previous study tackled both the olfaction and the risk of lateralization on the same candidates with such sample size comparing conchopexy to middle meatus spacer. Our study supports that the conchopexy, which is a very cost effective, is equivalent to the middle meatus spacer in preventing lateralization of the MTs and is not interfering with olfaction.

\section{Conclusions}

Conchopexy does not affect the olfaction subjectively. Neither conchopexy nor middle meatus spacer insertion was associated with olfaction loss among our cases. The number of patients who underwent MT lateralization with conchopexy was equal to that of those who underwent middle meatus spacer insertion. The olfaction improvement is related more to the underlying disease, as less improvement occurs in those with CRSsNP. Further prospective studies on the long-term outcome of conchopexy, its cost-effectiveness, and its impact on olfaction and the nasal airway using validated outcome tools are recommended.

\section{Additional Information}

\section{Disclosures}

Human subjects: All authors have confirmed that this study did not involve human participants or tissue. Animal subjects: All authors have confirmed that this study did not involve animal subjects or tissue. Conflicts of interest: In compliance with the ICMJE uniform disclosure form, all authors declare the following: Payment/services info: All authors have declared that no financial support was received from any organization for the submitted work. Financial relationships: All authors have declared that they have no financial relationships at present or within the previous three years with any organizations that might have an interest in the submitted work. Other relationships: All authors have declared that there are no other relationships or activities that could appear to have influenced the submitted work.

\section{References}

1. Moukarzel N, Nehmé A, Mansour S, Yammine FG, Moukheiber A: Middle turbinate medialization technique in functional endoscopic sinus surgery. J Otolaryngol. 2000, 29:144-147.

2. Thornton RS: Middle turbinate stabilization technique in endoscopic sinus surgery . Arch Otolaryngol Head Neck Surg. 1996, 8:869-872. 10.1001/archotol.1996.01890200059013

3. Friedman M, Tanyeri H, Landsberg R, Caldarelli D: Effects of middle turbinate medialization on olfaction . Laryngoscope. 1999, 9:1442-1445. 10.1097/00005537-199909000-00016

4. Bhalla RK, Kaushik V, de Carpentier J: Conchopexy suture to prevent middle turbinate lateralisation and septal haematoma after endoscopic sinus surgery. Rhinology. 2005, 43:143-145.

5. Hewitt KM, Orlandi RR: Suture medialization of the middle turbinates during endoscopic sinus surgery . Ear Nose Throat J. 2008, 87:E11.

6. Friedman M, Landsberg R, Tanyeri H: Middle turbinate medialization and preservation in endoscopic sinus surgery. Otolaryngol Head Neck Surg. 2000, 1:76-80. 10.1067/mhn.2000.105921

7. Lindemann J, Keck T, Rettinger G: Septal-turbinate-suture in endonasal sinus surgery. Rhinology. 2002, 40:92-94.

8. Friedman M, Schalch P: Middle turbinate medialization with bovine serum albumin tissue adhesive (BioGlue). Laryngoscope. 2008, 118:335-338. 10.1097/MLG.0b013e318158198f

9. Dutton JM, Hinton MJ: Middle turbinate suture conchopexy during endoscopic sinus surgery does not impair olfaction. Am J Rhinol Allergy. 2011, 2:125-127. 10.2500/ajra.2011.25.3560

10. Rettinger G, Lindemann K, Ashoor M, Scheithauer M, Sommer F, Lindemann J: Long term results of 


\section{Cureus}

transseptal suture of the middle turbinate during sinus surgery [Article in German]. Laryngo Rhino Otol. 2011, 8:471-475. 10.1055/s-0031-1280840

11. Hudson S, Orlandi R: Knot-free suture medialization of the middle turbinate. Int Forum Allergy Rhinol. 2013, 10:855-856. 10.1002/alr.21194

12. Bolger WE, Kuhn FA, Kennedy DW: Middle turbinate stabilization after functional endoscopic sinus surgery: the controlled synechiae technique. Laryngoscope. 1999, 109:1852-1853. 10.1097/00005537-19991100000025

13. Hegazy MA, Shawky A, El Fouly MS, El Kabani A: Conchopexy of middle turbinate versus bolgarization in endoscopic sinus surgery. Egypt J Otolaryngol. 2015, 31:219-223. 10.4103/1012-5574.168316

14. Chen W, Wang Y, Bi Y, Chen W: Turbinate-septal suture for middle turbinate medialization: a prospective randomized trial. Laryngoscope. 2015, 125:33-35. 10.1002/lary.24820

15. Hanna BM, Kilty SJ: Middle turbinate suture technique: a cost-saving and effective method for middle meatal preservation after endoscopic sinus surgery. J Otolaryngol Head Neck Surg. 2012, 41:407-412.

16. Bofares KM: Effect of middle turbinate intervention on outcomes of middle meatal endoscopic surgery . Pan Arab J Rhinol. 2016, 6:27-32. 10.4103/2090-7540.186905 